\title{
SCIENTIFIC REPORTS

\section{Total adiponectin is associated with incident cardiovascular and renal events in treated hypertensive patients: subanalysis of the ATTEMPT-CVD randomized trial}

\author{
Shokei Kim-Mitsuyama ${ }^{1 *}$, Hirofumi Soejima ${ }^{2,3}$, Osamu Yasuda ${ }^{4}$, Koichi Node ${ }^{5}{ }^{5}$, \\ Hideaki Jinnouchi ${ }^{6}$, Eiichiro Yamamoto ${ }^{2}$, Taiji Sekigami ${ }^{7}$, Hisao Ogawa ${ }^{8}$ \& Kunihiko Matsui ${ }^{9}$
}

The predictive value of serum adiponectin for hypertensive cardiovascular outcomes is unknown. This study was performed to investigate the association of adiponectin with incident cardiovascular and renal events ( $\mathrm{CV}$ events) in hypertensive patients. We performed post-hoc analysis on 1,228 hypertensive patients enrolled in the ATTEMPT-CVD study, a prospective randomized study comparing the effects of two antihypertensive therapies. The participants were divided into quartiles of baseline serum total adiponectin or high molecular weight (HMW) adiponectin. Multivariable Cox proportional hazards analysis was performed to determine the prognostic factors associated with $\mathrm{CV}$ events. Kaplan-Meier analysis for $\mathrm{CV}$ events by quartiles of baseline total adiponectin showed that patients in the highest total adiponectin quartile ( $Q 4)$ had more $C V$ events $(P=0.0135)$. On the other hand, no significant difference was noted regarding the incidence of $\mathrm{CV}$ events among patients stratified by $\mathrm{HMW}$ adiponectin quartile $(P=0.2551)$. Even after adjustment for potential confounders, the highest total adiponectin quartile $(\mathrm{Q} 4)$ remained independently associated with incident $\mathrm{CV}$ events in hypertensive patients $(\mathrm{HR}=1.949: 95 \% \mathrm{Cl} 1.051-3.612 ; \mathrm{P}=0.0341)$. These results showed that total adiponectin, but not $\mathrm{HMW}$ adiponectin, was independently associated with the incidence of $\mathrm{CV}$ events in treated hypertensive patients, thereby highlighting total adiponectin as a valuable predictor for hypertensive cardiovascular outcomes.

Adiponectin is the most abundant serum adipocytokine primarily secreted from adipose tissue and its serum concentrations decrease with obesity ${ }^{1-3}$. In vivo and in vitro experimental studies ${ }^{2-4}$ indicate that adiponectin exerts pleiotropic beneficial effects such as insulin-sensitizing, anti-inflammatory, anti-atherosclerotic, and anti cardioprotective effects. Low plasma adiponectin levels in humans are an indicator of the metabolic syndrome and associated with unfavourable cardiovascular and metabolic risk profile ${ }^{1,3,5,6}$, including insulin resistance, type 2 diabetes, low HDL-cholesterol, etc. Furthermore, low plasma adiponectin levels are significantly related to high risk of coronary artery disease in $\mathrm{men}^{7}$, an increased risk of mortality after ischemic stroke ${ }^{8}$, and neurological severity in ischemic stroke patients ${ }^{9}$. High plasma adiponectin concentrations are associated with lower risk of myocardial infarction in men $^{10}$ and are associated with a reduced risk for incident coronary heart disease events among men with type 2 diabetes ${ }^{11}$. Moreover, the meta-analysis also showed that higher adiponectin levels were associated with a low risk of coronary heart disease ${ }^{12}$. In contrast to the abundant evidence indicating inverse

\footnotetext{
${ }^{1}$ Department of Pharmacology and Molecular Therapeutics, Graduate School of Medical Sciences, Kumamoto University, Kumamoto, Japan. ${ }^{2}$ Department of Cardiovascular Medicine, Graduate School of Medical Sciences, Kumamoto University, Kumamoto, Japan. ${ }^{3}$ Health Care Center, Kumamoto University, Kumamoto, Japan. ${ }^{4}$ Department of Sports and Life Sciences, National Institute of Fitness and Sports in Kanoya, Kanoya, Japan. ${ }^{5}$ Department of Cardiovascular Medicine, Saga University, Saga, Japan. ${ }^{6}$ Diabetes Care Center, Jinnouchi Clinic, Kumamoto, Japan. ${ }^{7}$ Division of Internal Medicine \& Diabetes and Endocrine, Sekigami Clinic, Yatsushiro, Japan. ${ }^{8}$ National Cerebral and Cardiovascular Center, Suita, Japan. ${ }^{9}$ Department of General and Community Medicine, Kumamoto University Hospital, Kumamoto, Japan. *email: mitsuyam@gpo.kumamoto-u.ac.jp
} 
association of adiponectin levels in adverse cardiovascular outcomes, multiple lines of studies show conflicting findings proposing "adiponectin paradox" and indicate that high adiponectin concentrations are associated with adverse cardiovascular outcomes in various populations, such as patients with ischemic heart disease ${ }^{13,14}$, acute coronary syndrome $(\mathrm{ACS})^{15,16}$, chronic heart failure ${ }^{17-19}$, prevalent $\mathrm{CVD}^{20}$, chronic kidney disease $\mathrm{e}^{21}$, a relative young multi-ethnic population cohort ${ }^{22}$, or older adults ${ }^{23}$. Thus, the previous investigations show controversial findings regarding the association of adiponectin levels with cardiovascular outcomes.

In spite of the abundant reports on the association of adiponectin levels with various populations, little is known about the clinical value of circulating adiponectin levels in the pathophysiology of hypertension ${ }^{24-30}$. Most importantly, the association of adiponectin levels with CV outcomes in hypertensive patients remains to be determined. ATTEMPT-CVD study ${ }^{31,32}$ is a multicenter, prospective, randomized study to investigate the comparative effects of angiotensin receptor blocker (ARB)-based antihypertensive therapy versus non-ARB-based antihypertensive therapy on the change in various biomarkers and incident composite CV events in hypertensive outpatients with at least one cardiovascular risk. The prespecified biomarkers as the endpoint of ATTEMPT-CVD study included serum total adiponectin and serum high molecular weight (HMW) adiponectin ${ }^{31,32}$. Therefore, in the present study, to investigate the prognostic value of baseline serum total adiponectin and HMW adiponectin in hypertensive patients, we performed subanalysis on 1,228 hypertensive patients enrolled in the ATTEMMPT-CVD study.

\section{Results}

Baseline characteristics of patients categorized by quartile of serum total adiponectin. In the present study, serum total adiponectin levels at baseline were available for 1,228 patients enrolled in the ATTEMPT-CVD study. Table 1 shows baseline characteristics of patients categorized by quartile of serum total adiponectin at baseline. The median (25th-75th percentile) total adiponectin concentration was 5.28 (3.56-7.87) $\mu \mathrm{g} / \mathrm{mL}$. Patients with the highest total adiponectin levels in Q4 versus Q1, Q2, or Q3 were older $(\mathrm{P}<0.001)$, were less likely to be male $(\mathrm{P}<0.001)$, had lower $\mathrm{BMI}(\mathrm{P}=0.002)$, had lower diastolic $\mathrm{BP}(\mathrm{P}<0.001)$, were less likely to be current smoker $(\mathrm{P}<0.001)$, while, there was no difference among 4 groups of total adiponectin quartile with respect to systolic BP, proportion of diabetes mellitus, proportion of hyperlipidemia, proportion of previous cardiovascular disease, proportion of allocation to ARB based antihypertensive therapy.

Patients with total adiponectin levels in Q4 versus Q1, Q2, or Q3 had higher serum HMW adiponectin $(\mathrm{P}<0.001)$, higher HMW/total adiponectin ratio $(\mathrm{P}<0.001)$, higher plasma $\mathrm{BNP}(\mathrm{P}<0.001)$, lower eGFR $(\mathrm{P}<0.001)$, lower hsCRP $(\mathrm{P}<0.001)$, lower urinary 8 -OHdG $(\mathrm{P}=0.001)$, lower LDL-cholesterol $(\mathrm{P}<0.001)$, higher HDL-cholesterol $(\mathrm{P}<0.001)$, lower blood sugar $(\mathrm{P}=0.015)$, lower hemoglobin $\mathrm{A} 1 \mathrm{c}$ $(\mathrm{P}=0.001)$, and lower uric acid $(\mathrm{P}<0.001)$. While, there was no significant difference among patients with total adiponectin quartiles regarding $\operatorname{UACR}(\mathrm{P}=0.125)$.

Correlation of total adiponectin with other biomarkers. Supplementary Table 1 shows Spearman correlation coefficients between baseline serum total adiponectin and other biomarkers. Continuous serum total adiponectin concertation at baseline showed strong positive correlation with serum HMW adiponectin $(r=0.9756$, $\mathrm{P}<0.0001)$ and with $\mathrm{HMW} /$ total adiponectin ratio $(\mathrm{r}=0.8195, \mathrm{P}<0.0001)$. Serum total adiponectin exhibited weak positive correlation with age $(\mathrm{r}=0.3292, \mathrm{P}<0.0001)$ and plasma $\mathrm{BNP}(\mathrm{r}=0.3491, \mathrm{P}<0.0001)$, while exhibited very weak negative correlation with $\mathrm{BMI}(\mathrm{r}=-0.1107, \mathrm{P}=0.0001)$, eGFR $(\mathrm{r}=-0.1817, \mathrm{P}<0.0001)$, serum $\operatorname{hsCRP}(\mathrm{r}=-0.2179, \mathrm{P}<0.0001)$, urinary $8-\mathrm{OHdG}(\mathrm{r}=-0.1124, \mathrm{P}<0.0001), \mathrm{HbAlc}(\mathrm{r}=-0.07813, \mathrm{P}=0.0062)$, LDL-cholesterol $(r=-0.1156, P<0.0001)$, and uric acid $(r=-0.1706, P<0.0001)$. There was no correlation of serum total adiponectin with UACR $(\mathrm{P}=0.0541)$ or systolic $\mathrm{BP}(\mathrm{P}=0.3056)$.

Incidence of composite cardiovascular and renal events according to quartiles of serum total adiponectin. Figure 1 shows Kaplan-Meier curves for composite cardiovascular and renal events in patients by serum total adiponectin quartile. There was the significant difference in incident cardiovascular and renal events among total adiponectin quartiles $(\mathrm{P}=0.0135)$ and patients with total adiponectin concentration in $\mathrm{Q} 4$ had more cardiovascular and renal events. Detail of composite cardiovascular and renal events among patients in Q1-Q4 was shown in Table 2.

Blood pressure of patients with total adiponectin quartile during follow up period. During 3 years of follow up period, systolic BP was similar among total adiponectin quartiles (Supplementary Table 2). Although diastolic BP of patients with Q4 was slightly lower among total adiponectin quartiles during the follow up period, the difference was very small.

Incidence of composite cardiovascular and renal events according to quartile of serum HMW adiponectin or $\mathrm{HMW} /$ total adiponectin ratio. Differing from the case of total adiponectin, there was no significant difference in incidence of composite cardiovascular and renal events among patients with quartile of HMW adiponectin $(\mathrm{P}=0.2551)$ (Fig. 2(A)) or HMW/total adiponectin ratio $(\mathrm{P}=0.6533)$ (Fig. 2(B)).

Association of prognostic factors with composite cardiovascular and renal events. Table 3 shows results of the multivariable Cox regression analysis with the backward selection method to determine an adjusted hazard ratio of prognostic factor for composite cardiovascular and renal events. The original model included the following 11 covariates: total adiponectin Q2, total adiponectin Q3, total adiponectin Q4, sex, age, presence of previous cardiovascular diseases, presence of previous diabetes mellitus, UACR, plasma BNP, eGFR, and current smoking. Total adiponectin Q1 was used as the reference value (hazard ratio $=1$ ). In this analysis, age $(P=0.9692)$, eGFR $(P=0.0942)$, and current smoking $(P=0.0628)$ were not significantly associated with composite cardiovascular and renal events in overall patients, and hence, these covariates were deleted. On the 


\begin{tabular}{|c|c|c|c|c|c|}
\hline & \multicolumn{5}{|c|}{ Quartile of serum total adiponectin (Total adiponectin level $(\mu \mathrm{g} / \mathrm{mL})$ ) } \\
\hline & Q1 (0.84-3.56) & Q2 (3.57-5.28) & Q3 (5.29-7.87) & Q4 (7.88-41.59) & P value \\
\hline Number in each quartile & 308 & 308 & 306 & 306 & \\
\hline Age (years) & $62.7 \pm 9.5^{* *}$ & $64.7 \pm 9.9^{* *}$ & $67.6 \pm 8.6^{* *}$ & $70.2 \pm 7.7$ & $<0.001$ \\
\hline Male, n (\%) & $248(81)$ & $194(63)$ & $153(50)$ & $120(39)$ & $<0.001$ \\
\hline $\operatorname{BMI}\left(\mathrm{kg} / \mathrm{m}^{2}\right)$ & $25.6 \pm 3.4^{* *}$ & $25.6 \pm 3.9^{*}$ & $25.1 \pm 3.8$ & $24.7 \pm 4.2$ & 0.002 \\
\hline Systolic BP (mmHg) & $151.4 \pm 16.6$ & $150.5 \pm 15.7$ & $150.0 \pm 14.5$ & $149.9 \pm 16.0$ & 0.872 \\
\hline Diastolic BP (mmHg) & $86.7 \pm 11.4^{* *}$ & $85.1 \pm 11.9 * *$ & $83.7 \pm 11.1^{*}$ & $81.0 \pm 12.0$ & $<0.001$ \\
\hline Heart rate (b.p.m) & $72.9 \pm 11.0$ & $71.7 \pm 10.0$ & $71.6 \pm 11.6$ & $72.6 \pm 11.2$ & 0.508 \\
\hline Diabetes mellitus, $\mathrm{n}(\%)$ & $218(71)$ & $191(62)$ & $199(65)$ & $211(69)$ & 0.103 \\
\hline Hyperlipidemia, n (\%) & $193(63)$ & $178(58)$ & $167(55)$ & $166(54)$ & 0.122 \\
\hline Current smoker, $\mathrm{n}(\%)$ & $80(26)$ & $61(20)$ & $42(14)$ & $33(11)$ & $<0.001$ \\
\hline $\begin{array}{l}\text { Previous cardiovascular } \\
\text { disease, } \mathrm{n}(\%)\end{array}$ & $158(51.3)$ & $176(57.1)$ & $183(59.8)$ & $181(59.2)$ & 0.131 \\
\hline Allocation to ARB therapy, $\mathrm{n}(\%)$ & $142(46.1)$ & $162(52.6)$ & $160(52.3)$ & $151(49.3)$ & 0.335 \\
\hline HMW adiponectin $(\mu \mathrm{g} / \mathrm{mL})$ & $\begin{array}{l}0.86 * * \\
(0.62-1.14)\end{array}$ & $\begin{array}{l}1.84^{* *} \\
(1.57-2.16)\end{array}$ & \begin{tabular}{|l|}
$3.32 * *$ \\
$(2.75-3.87)$
\end{tabular} & $\begin{array}{l}6.44 \\
(5.24-8.83)\end{array}$ & $<0.001$ \\
\hline HMW/total adiponectin ratio & $0.31 \pm 0.11^{* *}$ & $0.43 \pm 0.09 * *$ & $0.51 \pm 0.09 * *$ & $0.62 \pm 0.09$ & $<0.001$ \\
\hline Plasma BNP (pg/mL) & \begin{tabular}{|l|}
$12.2 * *$ \\
$(6.2-21.9)$ \\
\end{tabular} & \begin{tabular}{|l|}
$16.6 * *$ \\
$(8.2-28.7)$ \\
\end{tabular} & \begin{tabular}{|l|}
$18.8^{* * *}$ \\
$(9.3-37.7)$ \\
\end{tabular} & $\begin{array}{l}31.2 \\
(16.9-61.0)\end{array}$ & $<0.001$ \\
\hline $\begin{array}{l}\text { UACR } \\
\text { (mg/g creatinine) }\end{array}$ & $\begin{array}{l}24.7 \\
(11.5-71.7)\end{array}$ & $\begin{array}{l}25.5 \\
(10.6-88.3)\end{array}$ & $\begin{array}{l}22.3 \\
(10.8-98.7)\end{array}$ & $\begin{array}{l}33.8 \\
(11.7-105.8)\end{array}$ & 0.125 \\
\hline $\begin{array}{l}\text { eGFR } \\
\left(\mathrm{ml} / \mathrm{min} \text { per } 1.73 \mathrm{~m}^{2}\right)\end{array}$ & \begin{tabular}{|l|}
$75.1 * *$ \\
$(65.0-88.7)$
\end{tabular} & $\begin{array}{l}72.2 * * \\
(61.7-85.7)\end{array}$ & $\begin{array}{l}68.5 \\
(57.2-83.8)\end{array}$ & $\begin{array}{l}67.5 \\
(55.8-78.8)\end{array}$ & $<0.001$ \\
\hline hsCRP (ng/mL) & $\begin{array}{l}793^{* *} \\
(413-1838)\end{array}$ & $\begin{array}{l}637 * * \\
(348-1188)\end{array}$ & \begin{tabular}{|l|}
$561^{* *}$ \\
$(283-1258)$
\end{tabular} & $\begin{array}{l}400 \\
(178-892)\end{array}$ & $<0.001$ \\
\hline Urinary 8-OHdG(ng/mL) & $\begin{array}{l}1.0 * * \\
(6.7-17.4)\end{array}$ & $\begin{array}{l}9.9 \\
(5.8-14.6)\end{array}$ & $\begin{array}{l}9.1 \\
(5.2-14.5)\end{array}$ & $\begin{array}{l}8.8 \\
(5.1-14.2)\end{array}$ & 0.001 \\
\hline \multicolumn{6}{|l|}{ Serum or plasma values } \\
\hline Creatinine $(\mathrm{mg} / \mathrm{dL})$ & $0.79 \pm 0.20$ & $0.78 \pm 0.23$ & $0.79 \pm 0.25$ & $0.79 \pm 0.26$ & 0.570 \\
\hline Potassium $(\mathrm{mEq} / \mathrm{L})$ & $4.26 \pm 0.48^{*}$ & $4.27 \pm 0.55$ & $4.30 \pm 0.46$ & $4.37 \pm 0.58$ & 0.029 \\
\hline Total cholesterol (mg/dL) & $197 \pm 36$ & $197 \pm 40$ & $196 \pm 34$ & $194 \pm 33$ & 0.677 \\
\hline LDL cholesterol (mg/dL) & $116 \pm 31^{* *}$ & $114 \pm 30^{*}$ & $112 \pm 28^{*}$ & $107 \pm 29$ & $<0.001$ \\
\hline HDL cholesterol (mg/dL) & $51 \pm 11 * *$ & $55 \pm 12 * *$ & $56 \pm 13^{* *}$ & $62 \pm 15$ & $<0.001$ \\
\hline Blood sugar $(\mathrm{mg} / \mathrm{dL})$ & $144 \pm 62 *$ & $132 \pm 52$ & $131 \pm 53$ & $132 \pm 56$ & 0.015 \\
\hline Hemoglobin Alc (\%) & $6.6 \pm 1.3^{* * *}$ & $6.3 \pm 1.2$ & $6.3 \pm 1.1$ & $6.3 \pm 1.1$ & 0.001 \\
\hline Uric acid (mg/dL) & $5.6 \pm 1.3^{* *}$ & $5.4 \pm 1.4^{* *}$ & $5.2 \pm 1.3$ & $5.1 \pm 1.3$ & $<0.001$ \\
\hline
\end{tabular}

Table 1. Baseline characteristics of patients categorized by quartile of serum total adiponectin. Abbreviations: $\mathrm{BMI}$, body mass index; $\mathrm{BP}$, blood pressure; $\mathrm{ARB}$, antihypertensive treatment with angiotensin II receptor blocker; HMW, high molecular weight; BNP, brain natriuretic peptide; UACR, urinary albumin/creatinine ratio; eGFR, estimated glomerular filtration rate; hsCRP, high sensitive C-reactive protein; 8-OHdG, 8-hydroxy2'-deoxyguanosine; LDL, low-density lipoprotein; HDL, high-density lipoprotein. HMW adiponectin, plasma BNP, UACR, eGFR, hsCRP and urinary $8-\mathrm{OHdG}$ are expressed as median with interquartile range. Other data are mean \pm s.d. for continuous values and number (\%) for categorical variables. P-value was calculated using Steel-Dwass or Tukey's multiple comparison test for continuous variables and $\chi^{2}$ test for categorical variables. $* \mathrm{P}<0.05, * * \mathrm{p}<0.01$ vs $\mathrm{Q} 4$.

other hand, total adiponectin concentration in Q4 was independently associated with composite cardiovascular and renal events $(\mathrm{HR}=1.949$ : 95\% CI 1.051-3.612; $\mathrm{P}=0.0341)$, while total adiponectin in $\mathrm{Q} 2(\mathrm{P}=0.6337)$ or $\mathrm{Q} 3(\mathrm{P}=0.7518)$ had no significant association with cardiovascular and renal events. Male gender $(\mathrm{P}=0.009)$, previous cardiovascular disease $(\mathrm{P}=0.0002)$, previous type 2 diabetes $(\mathrm{P}<0.0001), \mathrm{UACR} \geq 30 \mathrm{mg} / \mathrm{g}$ creatinine $(\mathrm{P}=0.0005)$, and plasma $\mathrm{BNP} \geq 19 \mathrm{mg} / \mathrm{ml}(\mathrm{P}=0.0053)$ were also significantly associated with cardiovascular and renal events.

\section{Discussion}

To our knowledge, the association of circulating adiponectin levels with cardiovascular and renal outcomes in hypertensive patients remains unknown. The major findings of our present study were that high total adiponectin was independently associated with incident cardiovascular and renal events in hypertensive patients receiving antihypertensive therapies and that in contrast to total adiponectin, HMW adiponectin had no significant association with cardiovascular and renal events. Thus, our findings suggest that total adiponectin, but not HMW adiponectin, may be a valuable predictor for incident cardiovascular and renal events in hypertensive patients.

Preclinical study shows that adiponectin-knockout mice exhibit the elevation of blood pressure and adiponectin supplementation significantly lowers blood pressure in hypertensive obese mice ${ }^{33}$. Therefore, adiponectin 


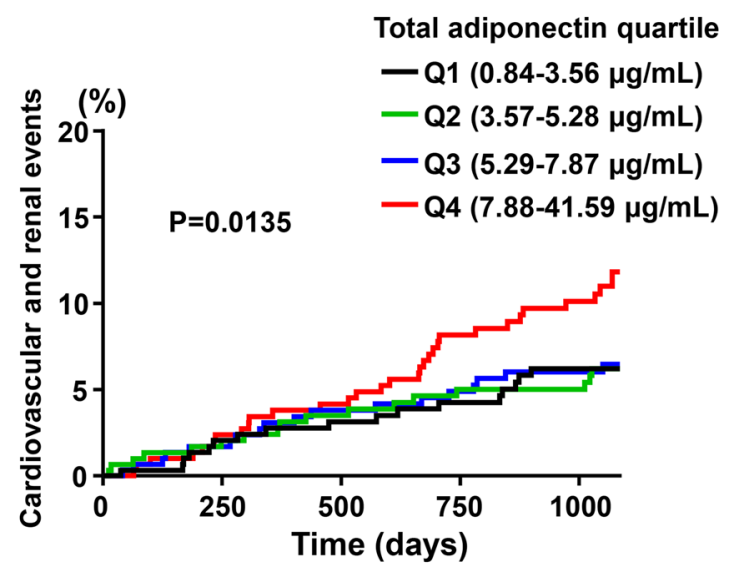

Figure 1. Kaplan-Meier curves for composite cardiovascular and renal events stratified by quartiles of serum total adiponectin at baseline. The number of occurrence of endpoints was 17, 18, 19, and 35 in Q1 ( $\mathrm{n}=306)$, Q2 $(\mathrm{n}=302), \mathrm{Q} 3(\mathrm{n}=303)$, and Q4 $(\mathrm{n}=302)$, respectively.

\begin{tabular}{|l|l|l|l|l|}
\hline \multirow{2}{*}{ Cardiovascular and renal events } & \multicolumn{4}{l}{ Quartile of total adiponectin } \\
\cline { 2 - 5 } & $\mathbf{Q 1}$ & $\mathbf{Q} 2$ & $\mathbf{Q 3}$ & $\mathbf{Q} 4$ \\
\cline { 2 - 5 } & $(\mathbf{n}=\mathbf{3 0 6})$ & $(\mathbf{n}=\mathbf{3 0 2})$ & $(\mathbf{n}=\mathbf{3 0 3})$ & $(\mathbf{n}=\mathbf{3 0 2})$ \\
\hline Total, $\mathrm{n}$ & 17 & 18 & 19 & 35 \\
\hline Stroke, $\mathrm{n}$ & 5 & 2 & 5 & 7 \\
\hline Transient ischemic attack, $\mathrm{n}$ & 1 & 1 & 1 & 0 \\
\hline Sudden death, $\mathrm{n}$ & 1 & 0 & 1 & 2 \\
\hline Acute myocardial infarction, $\mathrm{n}$ & 1 & 1 & 2 & 2 \\
\hline Angina pectoris, $\mathrm{n}$ & 0 & 1 & 2 & 3 \\
\hline Heart failure, $\mathrm{n}$ & 0 & 2 & 1 & 6 \\
\hline Aortic aneurysm, $\mathrm{n}$ & 2 & 0 & 0 & 1 \\
\hline Aortic dissection, $\mathrm{n}$ & 0 & 1 & 0 & 0 \\
\hline Peripheral artery disease, $\mathrm{n}$ & 3 & 2 & 2 & 3 \\
\hline Diabetic nephropathy, $\mathrm{n}$ & 1 & 1 & 0 & 0 \\
\hline Diabetic retinopathy, $\mathrm{n}$ & 3 & 2 & 3 & 5 \\
\hline Doubling of serum creatinine, $\mathrm{n}$ & 0 & 5 & 1 & 6 \\
\hline End stage renal disease, $\mathrm{n}$ & 0 & 0 & 1 & 0 \\
\hline
\end{tabular}

Table 2. Detail of composite cardiovascular and renal events among patients categorized by quartile of serum total adiponectin.

seems to play a protective role against hypertension. However, in clinical settings, the previous findings regarding the association of total adiponectin in the risk of hypertension are controversial ${ }^{24-30}$. Prospective studies in Chinese ${ }^{25}$ and Japanese ${ }^{26}$ populations and a systematic review and meta-analysis ${ }^{27}$ show that hypoadiponectinemia is significantly associated with high risk of hypertension. High total adiponectin levels are associated with reduced risk of incident hypertension ${ }^{29}$. On the other hand, other studies ${ }^{24,28,30}$ showed no association of total adiponectin with risk of hypertension. Thus, the predictive value of adiponectin in the incidence of hypertension remains to be defined. Furthermore, all previous studies ${ }^{24-30}$ focused on the association of adiponectin only with incidence of hypertension, and have not examined the association with the incidence of hypertensive adverse cardiovascular outcomes. Thus, at present, the association of adiponectin levels with cardiovascular and renal events in hypertensive patients is unknown. These findings encouraged us to investigate the association of baseline adiponectin levels with cardiovascular and renal events among hypertensive patients enrolled in the ATTEMPT-CVD study.

It is an important issue whether potential difference in BP among quartiles of total adiponectin might contribute to the higher incidence of cardiovascular and renal events in the highest total adiponectin quartile (Q4), since ATTEMPT-CVD study was designed to compare the effect of two antihypertensive therapies and adiponectin provokes antihypertensive effect evidenced by animal study ${ }^{33}$. However, in the present analysis, systolic BP at baseline and during antihypertensive treatment was comparable among patients stratified by quartile of total adiponectin. Moreover, patients in the highest total adiponectin quartile (Q4) had slightly lower diastolic BP at baseline and during antihypertensive treatment, although the difference was very small. Thus, BP seems not to be related to higher incidence of cardiovascular and renal events in the highest total adiponectin quartile (Q4).

Circulating adiponectin levels are known to be affected by various factors, such as plasma BNP, renal function, aging and $\operatorname{sex}^{2,34-36}$. In the present study, increasing total adiponectin from Q1 to Q4 was associated with the 
(A)

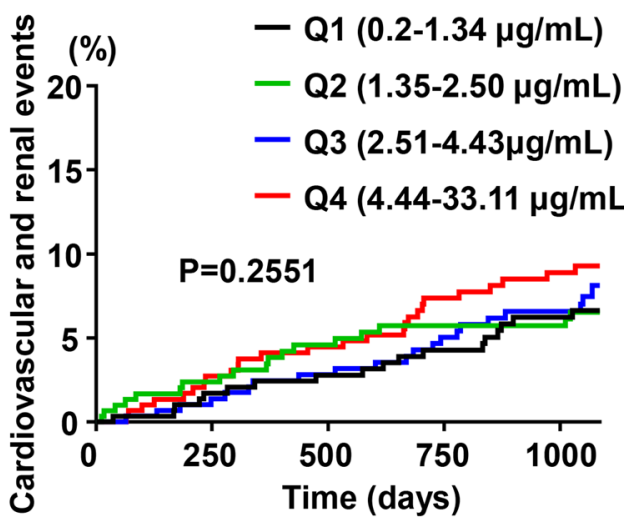

(B)

HMW/Total adiponectin ratio

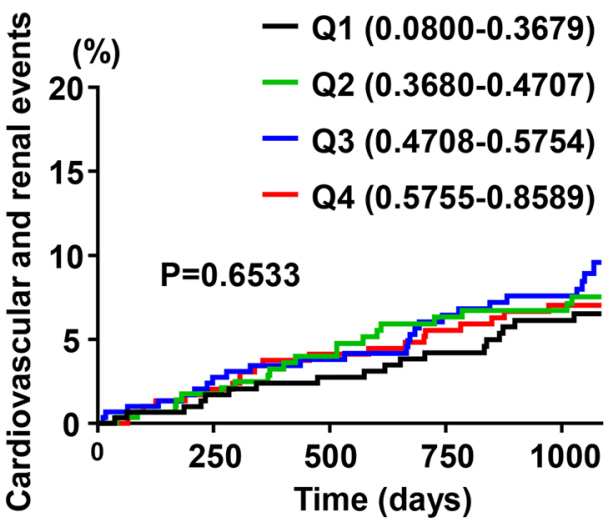

Figure 2. Kaplan-Meier curves for composite cardiovascular and renal events stratified by quartiles of serum HMW adiponectin (A) or by quartiles of HMW/total adiponectin ratio (B). (A) The number of occurrence of endpoints was 18,19,22, and 30 in Q1 $(n=306)$, Q2 $(n=303)$, Q3 $(n=301)$, and Q4 $(n=303)$, respectively. (B) The number of occurrence of endpoints was 18, 21, 25, and 25 in Q1 $(n=306), Q 2(n=303), Q 3(n=300)$, and $\mathrm{Q} 4(\mathrm{n}=304)$, respectively.

\begin{tabular}{|l|l|l|}
\hline & HR $(\mathbf{9 5} \% \mathbf{C I})$ & P-value \\
\hline Total adiponectin Q1 (Reference) & 1 & \\
\hline Total adiponectin Q2 & $1.177(0.602-2.302)$ & 0.6337 \\
\hline Total adiponectin Q3 & $1.114(0.570-2.177)$ & 0.7518 \\
\hline Total adiponectin Q4 & $1.949(1.051-3.612)$ & 0.0341 \\
\hline Male gender & $1.851(1.166-2.936)$ & 0.0090 \\
\hline Previous cardiovascular disease & $2.521(1.541-4.123)$ & 0.0002 \\
\hline Previous type 2 diabetes & $3.077(1.762-5.375)$ & $<0.0001$ \\
\hline UACR $\geq 30 \mathrm{mg} / \mathrm{g}$ creatinine & $2.290(1.438-3.645)$ & 0.0005 \\
\hline Plasma BNP $\geq 19 \mathrm{mg} / \mathrm{ml}$ & $1.921(1.214-3.040)$ & 0.0053 \\
\hline
\end{tabular}

Table 3. Adjusted hazard ratios of prognostic factor for composite cardiovascular and renal events in overall patients by multivariable Cox proportional analysis with the backward selection method. The original model included the following 11 covariates: Total adiponectin Q2, Total adiponectin Q3, Total adiponectin Q4, sex, age, presence of baseline cardiovascular diseases, presence of baseline type 2 diabetes, UACR, plasma BNP, eGFR, and current smoking. Of the 11 covariates, age $(\mathrm{P}=0.9692)$, eGFR $(\mathrm{P}=0.0942)$, and current smoking $(\mathrm{P}=0.0628)$ had no significant association with composite cardiovascular and renal events in overall patients, and therefore these covariates were deleted. Abbreviations: HR, hazard ratio; 95\%CI, 95\% confidence interval; $\mathrm{UACR}$, urinary albumin/creatinine ratio; BNP, brain natriuretic peptide. Total adiponectin concentration in Q1 was used as the reference $(\mathrm{HR}=1.0)$.

increased age, more female proportion, and increased plasma BNP, while associated with the decreased eGFR. In the present study, we have carried out multivariable Cox proportional hazards analysis with the backward selection starting with 11 covariates including age, sex, plasma BNP, and eGFR, etc. Of note, even after adjustment for potential confounders, the association of the highest total adiponectin quartile (Q4) with composite cardiovascular and renal events remained significant. Therefore, our study showed that high total adiponectin is independently associated with the incidence of cardiovascular and renal events in hypertensive patients.

Adiponectin in human serum exists as a trimer, a hexamer, and HMW form ${ }^{34,37}$. Importantly, HMW adiponectin is regarded to be the most active form, and is more associated with insulin sensitivity and with lower risk for incident diabetes than total adiponectin ${ }^{38,39}$, and confers more protection against endothelial cell apoptosis ${ }^{40}$. Based on these findings, it has been proposed that HMW adiponectin concentrations and HMW/total adiponectin ratio ${ }^{37,41}$ may be more useful biomarker than total adiponectin for evaluating risk of cardiovascular and renal diseases. However, almost previous studies regarding the association of adiponectin with cardiovascular outcomes among various populations have been limited to total adiponectin. Limited numbers of studies regarding HMW adiponectin have been reported and showed inconsistent findings on the association with cardiovascular outcomes ${ }^{19,37,38,42-44}$. Moreover, the association of HMW adiponectin or HMW/total adiponectin ratio with hypertensive cardiovascular events has not been reported. In the present study, we found strong positive correlation of total adiponectin with HMW adiponectin and HMW/total adiponectin ratio. However, in contrast to the significant association of total adiponectin with cardiovascular and renal events, neither HMW adiponectin 
nor HMW / total adiponectin ratio was associated with the incidence of cardiovascular and renal events in hypertensive patients. Therefore, our present findings suggest that total adiponectin may be more useful predictor for hypertensive cardiovascular and renal events than HMW adiponectin.

The present study did not allow us to elucidate the mechanism and the significance of high circulating adiponectin levels in the Q4 group of hypertensive patients. It has been proposed that high circulating adiponectin levels in various populations such as heart failure, ischemic heart disease or CKD etc. might be attributed to counter-regulatory upregulation of adiponectin production in response to various stresses caused by such severe chronic diseases ${ }^{2,13-15,17,21}$. Accumulating experimental evidence indicates that adiponectin has pleiotrophic beneficial effects including anti-inflammatory, antiatherogenic, or cardioprotective actions $s^{3,45-47}$. Therefore, it is likely that high circulating adiponectin levels in the Q4 group of high-risk hypertensive patients might be at least partially explained by compensatory upregulation of adiponectin production in response to severe chronic stress related to hypertension. However, animal and clinical studies show that functional adiponectin resistance develops in various chronic diseases such as obesity, diabetes mellitus or heart failure ${ }^{2,45,48-51}$. Previous reports ${ }^{50,51}$ show that the increased circulating adiponectin levels in patients with heart failure are accompanied by the downregulation of its adiponectin receptor and decreased downstream signaling such as deactivation of the PPAR- $\alpha /$ AMPK pathway and downregulation of several target genes in skeletal muscles, thereby proposing the failure of adiponectin to exert significant beneficial effects (functional adiponectin resistance) in such chronic disease. Therefore, it remains uncertain whether high adiponectin levels in Q4 quartile might significantly exert cardiovascular protective effect. Alternatively, in vitro study ${ }^{52}$ indicates that adiponectin binds $\mathrm{C} 1 \mathrm{q}$ and activates the classical pathway of complement, thereby suggesting that adiponectin may exert pro-inflammatory action rather than anti-inflammatory action. Therefore, it cannot be completely excluded that high adiponectin levels in the Q4 group might be involved in the progression of hypertensive CV events through pro-inflammatory action ${ }^{2,52}$. Further study is required to elucidate the mechanism for such compensatory response of adiponectin and its protective or detrimental role in cardiovascular diseases in the clinical setting.

Study limitation. There are several limitations in this study. First, the present study was a post-hoc analysis of ATTEMPT-CVD study, although serum total adiponectin and HMW adiponectin were prespecified as a secondary endpoint of the ATTEMPT-CVD study. Second, the present study did not allow us to elucidate the causal relationship between high total adiponectin and the increased cardiovascular and renal events in hypertensive patients. Third, in the present study, increasing total adiponectin levels from Q1 to Q4 led to beneficial cardiovascular profile as shown by the decrease in diastolic BP, inflammatory marker (hs-CRP), oxidative stress marker (8-OHdG), HbA1c, blood sugar, and LDL-cholesterol and the increase in HDL-cholesterol. It is unclear whether such beneficial cardiovascular profile in high total adiponectin quartile might be attributed to compensatory mechanism mediated by pleiotrophic effects of adiponectin, although the beneficial effects of adiponectin shown by experimental studies might be partially involved in such beneficial cardiovascular profile. Fourth, it cannot be completely excluded that unknown confounding factor(s) might affect our present findings, although the association of total adiponectin with cardiovascular and renal events remained significant even after adjustment for potential confounders. Fifth, although circulating adiponectin derives primarily from adipose tissue, it is also produced by other types of cells including cardiomyocytes, skeletal muscles and endothelial cells. However, it remains uncertain whether adiponectin from non-adipose tissue significantly contributed to high adiponectin levels in Q4 quartile. Finally, the underlying mechanism of differential impacts of total and HMW adiponectin on cardiovascular outcomes in hypertension is not elucidated in this study.

In conclusion, we obtained the evidence that total adiponectin, but not HMW adiponectin, was independently associated with the incidence of cardiovascular and renal events in hypertensive patients under antihypertensive therapies. Thus, we propose that total adiponectin may be a useful predictor for incident cardiovascular and renal events in hypertensive patients. However, larger scale prospective study is required to define the precise value of total adiponectin and HMW adiponectin as a predictive marker for hypertensive cardiovascular and renal outcomes.

\section{Patients and Methods}

The inclusion and exclusion criteria, and study protocol of ATTEMPT-CVD. The study design ${ }^{32}$ and the primary results ${ }^{31}$ of ATTEMPT-CVD study (ClinicalTrials. gov number NCT01075698) have been previously published.

The ATTEMPT-CVD study was a multicenter, prospective, randomized open label, controlled trial to compare the effect of an angiotensin receptor blocker (ARB)(telmisartan)-based antihypertensive therapy vs non-ARB antihypertensive therapy on various biomarkers and composite cardiovascular and renal events in hypertensive outpatients ${ }^{31,32}$. Patients were followed up for three years at 168 institutions throughout Japan. Patients were eligible if they were 40-79-year-old hypertensive outpatients who had at least one cardiovascular risk (type 2 diabetes, renal factors, cardiac factors, cerebral factors and peripheral arterial factors). The exclusion criteria included type 1 diabetes, severe renal disorder (serum creatinine level $\geq 2.0 \mathrm{mg} / \mathrm{dL}$ ) and the occurrence of heart failure NYHA Classification III or IV, myocardial infarction, percutaneous revascularization and bypass grafting of coronary artery/lower extremity vessel, cerebral infarction, cerebral hemorrhage, subarachnoid hemorrhage and transient cerebral ischemic attack within 6 months before the observation period, and it also included malignant hypertension, secondary hypertension, pregnant women, clinically problematic allergic disease or past history of hypersensitivity to the drugs used, past history of significant adverse drug reactions, extremely poor biliary secretion or serious hepatic disorder, patients who require treatment for a malignant tumor, and other patients who are judged by the physician to be unsuitable for the study. Full inclusion and exclusion criteria are described in our protocol paper ${ }^{32}$. All participants provided written informed consent. The study protocol is in agreement with the guidelines of the ethics committees at our institutions and the study complies with the Declaration of 
Helsinki. The institutional review board of each participating hospital approved this trial (See Appendix A), and written informed consent was obtained from each patient. The study protocol was approved by Independent Ethics Committee of Kumamoto University.

The physicians examined the survey items at the start of the study (at registration), after 3, 6, 12, 24 and 36 months from the start of the study, at a discontinuation/dropout, at the occurrence of any cardiovascular and renal event, and at the occurrence of any adverse event.

Endpoints of ATTEMPT-CVD study. In ATTEMPT-CVD study, the primary endpoints were changes in urinary albumin/creatinine ratio (UACR) and changes in plasma BNP levels from baseline, as described. The incidence of composite cardiovascular and renal events was a prespecified secondary endpoint of ATTMEPT-CVD study. The composite cardiovascular and renal events included cerebral events (cerebral infarction, cerebral hemorrhage, subarachnoid hemorrhage, unknown type of stroke, transient ischemic attack), cardiac events (sudden death, myocardial infarction, angina pectoris, asymptomatic myocardial ischemia, heart failure), aortic/peripheral arterial events (aortic aneurysm, aortic dissection, arteriosclerotic disease), newly occurred (or) aggravated diabetic complications (diabetic nephropathy, diabetic retinopathy, diabetic neuropathy), aggravation of renal function (doubling of serum creatinine level, initiation of dialysis, renal transplantation).

In addition, the changes in serum total adiponectin, serum high molecular weight (HMW) adiponectin, plasma high sensitive C-reactive protein (hsCRP), urinary 8-hydroxy-2'-deoxyguanosine (8-OHdG), and estimated glomerular filtration rate (eGFR) were also specified as the secondary endpoints of ATTEMPT-CVD study.

Measurement of adiponectin and other biomarkers. The plasma or serum concentrations of all biomarkers were measured in SRL, Inc. (Tokyo, Japan). Serum total adiponectin and HMW adiponectin concentrations were measured by ELISA kit (Sekisui Medical Co., LTD., Tokyo, Japan)

Statistical analyses. All analyses were performed on the intention-to-treat population. Serum total adiponectin concentration, HMW adiponectin concentration, and HMW/total adiponectin ratio at baseline were categorized by quartiles (Q1, Q2, Q3, Q4). As for cardiovascular and renal events, time to first event curves were estimated by the Kaplan-Meier method and the log-rank test was used to analyze the differences among patients with quartiles (Q1, Q2, Q3, Q4) of serum total adiponectin, HMW adiponectin, or HMW/total adiponectin ratio. Using Cox proportional hazard model, the hazard ratio (HR) of Q1-Q4 and its 95\% confidence interval (CI) were calculated. Two-way repeated measures analysis of variance was used to compare among Q1-Q4 for time course of systolic or diastolic blood pressure during the follow-up period. Multivariable Cox proportional hazards analysis combined with the backward selection method was performed to determine the association of total adiponectin with the incidence of composite cardiovascular and renal events independently. The original model included the following 11 covariates: total adiponectin in Q2, Q3, and Q4, sex, age, presence of baseline cardiovascular diseases, presence of baseline type 2 diabetes, eGFR, plasma BNP, UACR, and current smoking. Total adiponectin in Q1 was used as the reference value and its HR was expressed as 1 . To compare baseline parameters among patients in Q1-Q4, P-value was calculated using Steel-Dwass or Tukey's multiple comparison test for continuous variables and $\chi^{2}$ test for categorical variables. Spearman correlation coefficients were calculated between total adiponectin concentration and others biomarkers at baseline. Windows SAS Version 9.2 and subsequent versions were used as the statistical analysis software. P-values of less than 0.05 were considered statistically significant.

\section{Data availability}

The datasets generated during and/or analysed during the current study are available from the corresponding author on reasonable request.

Received: 19 July 2019; Accepted: 23 October 2019;

Published online: 12 November 2019

\section{References}

1. Ryo, M. et al. Adiponectin as a biomarker of the metabolic syndrome. Circ J 68, 975-981 (2004).

2. Kizer, J. R. Adiponectin, cardiovascular disease, and mortality: parsing the dual prognostic implications of a complex adipokine. Metabolism 63, 1079-1083 (2014).

3. Turer, A. T. \& Scherer, P. E. Adiponectin: mechanistic insights and clinical implications. Diabetologia 55, 2319-2326 (2012).

4. Shibata, R. et al. Adiponectin protects against myocardial ischemia-reperfusion injury through AMPK- and COX-2-dependent mechanisms. Nat Med 11, 1096-1103 (2005).

5. Santaniemi, M., Kesaniemi, Y. A. \& Ukkola, O. Low plasma adiponectin concentration is an indicator of the metabolic syndrome. Eur J Endocrinol 155, 745-750 (2006).

6. Weyer, C. et al. Hypoadiponectinemia in obesity and type 2 diabetes: close association with insulin resistance and hyperinsulinemia. J Clin Endocrinol Metab 86, 1930-1935 (2001).

7. Kumada, M. et al. Association of hypoadiponectinemia with coronary artery disease in men. Arterioscler Thromb Vasc Biol 23, 85-89 (2003).

8. Efstathiou, S. P. et al. Plasma adiponectin levels and five-year survival after first-ever ischemic stroke. Stroke 36, 1915-1919 (2005).

9. Kuwashiro, T. et al. Significance of plasma adiponectin for diagnosis, neurological severity and functional outcome in ischemic stroke - Research for Biomarkers in Ischemic Stroke (REBIOS). Metabolism 63, 1093-1103 (2014).

10. Pischon, T. et al. Plasma adiponectin levels and risk of myocardial infarction in men. JAMA 291, 1730-1737 (2004).

11. Schulze, M. B. et al. Adiponectin and future coronary heart disease events among men with type 2 diabetes. Diabetes 54, 534-539 (2005).

12. Zhang, H. et al. Adiponectin levels and risk of coronary heart disease: a meta-analysis of prospective studies. Am J Med Sci 345, 455-461 (2013).

13. Beatty, A. L. et al. Adiponectin is associated with increased mortality and heart failure in patients with stable ischemic heart disease: data from the Heart and Soul Study. Atherosclerosis 220, 587-592 (2012). 
14. Cavusoglu, E. et al. Adiponectin is an independent predictor of all-cause mortality, cardiac mortality, and myocardial infarction in patients presenting with chest pain. Eur Heart J 27, 2300-2309 (2006).

15. Bergmark, B. A. et al. Baseline adiponectin concentration and clinical outcomes among patients with diabetes and recent acute coronary syndrome in the EXAMINE trial. Diabetes Obes Metab 19, 962-969 (2017).

16. Wilson, S. R. et al. Assessment of adiponectin and the risk of recurrent cardiovascular events in patients presenting with an acute coronary syndrome: observations from the Pravastatin Or atorVastatin Evaluation and Infection Trial-Thrombolysis in Myocardial Infarction 22 (PROVE IT-TIMI 22). Am Heart J 161, 1147-1155 e1141 (2011).

17. Kistorp, C. et al. Plasma adiponectin, body mass index, and mortality in patients with chronic heart failure. Circulation 112, 1756-1762 (2005).

18. Lindberg, S. et al. Cardio-adipose tissue cross-talk: relationship between adiponectin, plasma pro brain natriuretic peptide and incident heart failure. Eur J Heart Fail 16, 633-638 (2014).

19. Tsutamoto, T. et al. Total and high molecular weight adiponectin, haemodynamics, and mortality in patients with chronic heart failure. Eur Heart J 28, 1723-1730 (2007).

20. Dekker, J. M. et al. Prognostic value of adiponectin for cardiovascular disease and mortality. J Clin Endocrinol Metab 93, 1489-1496 (2008).

21. Menon, V. et al. Adiponectin and mortality in patients with chronic kidney disease. J Am Soc Nephrol 17, 2599-2606 (2006).

22. Witberg, G. et al. Relation of Adiponectin to All-Cause Mortality, Cardiovascular Mortality, and Major Adverse Cardiovascular Events (from the Dallas Heart Study). Am J Cardiol 117, 574-579 (2016).

23. Poehls, J. et al. Association of adiponectin with mortality in older adults: the Health, Aging, and Body Composition Study. Diabetologia 52, 591-595 (2009).

24. Asferg, C. et al. Leptin, not adiponectin, predicts hypertension in the Copenhagen City Heart Study. Am J Hypertens 23, 327-333 (2010).

25. Chow, W. S. et al. Hypoadiponectinemia as a predictor for the development of hypertension: a 5-year prospective study. Hypertension 49, 1455-1461 (2007).

26. Imatoh, T., Miyazaki, M., Momose, Y., Tanihara, S. \& Une, H. Adiponectin levels associated with the development of hypertension: a prospective study. Hypertens Res 31, 229-233 (2008).

27. Kim, D. H., Kim, C., Ding, E. L., Townsend, M. K. \& Lipsitz, L. A. Adiponectin levels and the risk of hypertension: a systematic review and meta-analysis. Hypertension 62, 27-32 (2013).

28. Onat, A. et al. High adiponectin levels fail to protect against the risk of hypertension and, in women, against coronary disease: involvement in autoimmunity? World J Diabetes 4, 219-225 (2013).

29. Peri-Okonny, P. A. et al. Adiponectin protects against incident hypertension independent of body fat distribution: observations from the Dallas Heart Study. Diabetes Metab Res Rev 33 (2017).

30. Seven, E. et al. Overweight, adipocytokines and hypertension: a prospective population-based study. J Hypertens 32, 1488-1494, discussion 1494 (2014).

31. Ogawa, H. et al. A trial of telmisartan prevention of cardiovascular diseases (ATTEMPT-CVD): Biomarker study. Eur J Prev Cardiol 23, 913-921 (2016).

32. Soejima, H. et al. The Changes of Biomarkers by Telmisartan and their Significance in Cardiovascular Outcomes: Design of a Trial of Telmisartan Prevention of Cardiovascular Diseases (ATTEMPT-CVD). J Clin Trials 4, 162 (2014).

33. Ohashi, K. et al. Adiponectin replenishment ameliorates obesity-related hypertension. Hypertension 47, 1108-1116 (2006).

34. Nakano, Y. et al. A novel enzyme-linked immunosorbent assay specific for high-molecular-weight adiponectin. J Lipid Res 47, 1572-1582 (2006).

35. Nishizawa, H. et al. Androgens decrease plasma adiponectin, an insulin-sensitizing adipocyte-derived protein. Diabetes 51, 2734-2741 (2002).

36. Tsukamoto, O. et al. Natriuretic peptides enhance the production of adiponectin in human adipocytes and in patients with chronic heart failure. J Am Coll Cardiol 53, 2070-2077 (2009).

37. Aso, Y. et al. Comparison of serum high-molecular weight (HMW) adiponectin with total adiponectin concentrations in type 2 diabetic patients with coronary artery disease using a novel enzyme-linked immunosorbent assay to detect HMW adiponectin. Diabetes 55, 1954-1960 (2006).

38. Nakashima, R. et al. Decreased total and high molecular weight adiponectin are independent risk factors for the development of type 2 diabetes in Japanese-Americans. J Clin Endocrinol Metab 91, 3873-3877 (2006).

39. Hara, K. et al. Measurement of the high-molecular weight form of adiponectin in plasma is useful for the prediction of insulin resistance and metabolic syndrome. Diabetes Care 29, 1357-1362 (2006).

40. Kobayashi, H. et al. Selective suppression of endothelial cell apoptosis by the high molecular weight form of adiponectin. Circ Res 94, e27-31 (2004)

41. Pajvani, U. B. et al. Complex distribution, not absolute amount of adiponectin, correlates with thiazolidinedione-mediated improvement in insulin sensitivity. J Biol Chem 279, 12152-12162 (2004).

42. Kizer, J. R. et al. Associations of total and high-molecular-weight adiponectin with all-cause and cardiovascular mortality in older persons: the Cardiovascular Health Study. Circulation 126, 2951-2961 (2012).

43. Sattar, N. et al. High molecular weight adiponectin is not associated with incident coronary heart disease in older women: a nested prospective case-control study. J Clin Endocrinol Metab 93, 1846-1849 (2008).

44. Karas, M. G. et al. Relations of plasma total and high-molecular-weight adiponectin to new-onset heart failure in adults $>/=65$ years of age (from the Cardiovascular Health study). Am J Cardiol 113, 328-334 (2014).

45. Lau, W. B. et al. Role of Adipokines in Cardiovascular Disease. Circ J 81, 920-928 (2017).

46. Szmitko, P. E., Teoh, H., Stewart, D. J. \& Verma, S. Adiponectin and cardiovascular disease: state of the art? Am J Physiol Heart Circ Physiol 292, H1655-1663 (2007).

47. Tilg, H. \& Moschen, A. R. Adipocytokines: mediators linking adipose tissue, inflammation and immunity. Nat Rev Immunol 6, 772-783 (2006).

48. Lin, H. V. et al. Adiponectin resistance exacerbates insulin resistance in insulin receptor transgenic/knockout mice. Diabetes 56, 1969-1976 (2007).

49. Mullen, K. L. et al. Adiponectin resistance precedes the accumulation of skeletal muscle lipids and insulin resistance in high-fat-fed rats. Am J Physiol Regul Integr Comp Physiol 296, R243-251 (2009).

50. Van Berendoncks, A. M. et al. Functional adiponectin resistance at the level of the skeletal muscle in mild to moderate chronic heart failure. Circ Heart Fail 3, 185-194 (2010).

51. Van Berendoncks, A. M. et al. Exercise training reverses adiponectin resistance in skeletal muscle of patients with chronic heart failure. Heart 97, 1403-1409 (2011).

52. Peake, P. W., Shen, Y., Walther, A. \& Charlesworth, J. A. Adiponectin binds $\mathrm{Clq}$ and activates the classical pathway of complement. Biochem Biophys Res Commun 367, 560-565 (2008). 


\section{Acknowledgements}

This work was supported by a grant from Japan Foundation for Aging and Health. We thank all ATTEMPT-CVD investigators who contributed to data collection, and patients, physicians, and medical staff who supported this clinical study. We also thank Kazuko Noda and Tomoko Moriyama for their excellent support during this study.

\section{Author contributions}

H.O., S.K.-M., K.N., H.J. and T.S. were steering committee members of this clinical trial, conducted this trial, performed data collection and interpretation, and contributed to the writing of the manuscript. K.M. conducted this trial, performed data collection, analysis, and interpretation, was a study statistician, and also contributed to the preparation of the manuscript. H.S., O.Y. and E.Y. conducted the trial performed data collection, data analysis, and data interpretation, and also contributed to the preparation of the manuscript.

\section{Competing interests}

Shokei Kim-Mitsuyama has received consultancy fees/honoraria/research grant from Astellas, DaiichiSankyo, and Takeda. Osamu Yasuda has received consultancy fees/honoraria/research grant from Otsuka Pharmaceutical Co., Ltd and Sanwa Kagaku Kenkyusho Co., Ltd. Koichi Node has received consultancy fees/ honoraria/research grant from Boerhinger Ingelheim. Hideaki Jinnouchi has received consultancy fees/ honoraria/research grant from AstraZeneca Pharmaceuticals, Astellas Pharma, Boehringer Ingelheim, Daiichi-sankyo, Eli Lilly, Takeda, Novartis Pharmaceuticals, Novo Nordisk and Sanofi. Kunihiko Matsui has received consultancy fees/honoraria/research grant from Daiici-Sankyo, Japan Boehringer-Ingelheim. All other coauthors declare that they have no financial competing interests. All authors declare that they have no nonfinancial competing interests.

\section{Additional information}

Supplementary information is available for this paper at https://doi.org/10.1038/s41598-019-52977-x.

Correspondence and requests for materials should be addressed to S.K.-M.

Reprints and permissions information is available at www.nature.com/reprints.

Publisher's note Springer Nature remains neutral with regard to jurisdictional claims in published maps and institutional affiliations.

(c) (i) Open Access This article is licensed under a Creative Commons Attribution 4.0 International License, which permits use, sharing, adaptation, distribution and reproduction in any medium or format, as long as you give appropriate credit to the original author(s) and the source, provide a link to the Creative Commons license, and indicate if changes were made. The images or other third party material in this article are included in the article's Creative Commons license, unless indicated otherwise in a credit line to the material. If material is not included in the article's Creative Commons license and your intended use is not permitted by statutory regulation or exceeds the permitted use, you will need to obtain permission directly from the copyright holder. To view a copy of this license, visit http://creativecommons.org/licenses/by/4.0/.

(c) The Author(s) 2019 